\title{
Soft-Error Performance Evaluation on Emerging Low Power Devices
}

\author{
Huichu Liu, Student Member, IEEE, Matthew Cotter, Student Member, IEEE, \\ Suman Datta, Fellow, IEEE, and Vijaykrishnan Narayanan, Fellow, IEEE
}

\begin{abstract}
Radiation-induced single-event upset (SEU) has become a key challenge for cloud computing. The proposed introduction of low bandgap materials (Ge, III-Vs) as channel replacement and steep switching devices for low-voltage applications may induce radiation reliability issues due to their low ionization energy and device architecture. In this paper, the softerror generation and propagation in Si FinFET, III-V FinFET, and III-V Hetero-junction tunnel FET (HTFET) are investigated using device and circuit simulation. III-V FinFET shows enhanced charge collection compared with Si FinFET, whereas HTFET shows significant reduction of the bipolar gain effect and charge collection. Soft-error rate (SER) evaluation methodology has been proposed for these emerging devices based on the critical LET extraction. SRAM bit flip, electrical masking effect, and latching window masking effect have been analyzed with supply voltage scaling. The SER evaluation of SRAM and logic shows that HTFET-based circuits are promising for radiation resilient ultralow power applications. III-V FinFET shows increased SER for SRAM for $V_{D D}$ range of 0.3-0.8 Vand reduced logic SER below 0.5 V compared with Si FinFET.
\end{abstract}

Index Terms-Heterojunction tunnel FET (HTFET), III-V FinFET, Bipolar gain effect, soft-error rate (SER), SRAM bit-flip, electrical masking, latching window masking.

\section{INTRODUCTION}

W ITH growing numbers of processors in data centers and constrained power budgets for high-end computation, radiation-induced single-event upset (SEU) has become a key challenge for cloud computing these days. According to the reported experiment results [1], the soft-error rate (SER) per logic state bit increases $8 \%$ for each technology generation. With the number of state bits per chip doubling at each generation, the expected SER per chip will have 100 times increase from $180 \mathrm{~nm}$ to $16 \mathrm{~nm}$ technology node [2], which can result in a faster-increasing trend of total data center SER (Fig. 1). Another key limit for today's system design is the power consumption budget in silicon CMOS technology. For low power applications these days, the near threshold computing as well

Manuscript received June 4, 2013; revised December 25, 2013 and March 1, 2014; accepted March 24, 2014. Date of publication April 10, 2014; date of current version June 3, 2014. This work was supported in part by Intel Academic Research Office (ARO) and in part by the U.S. National Science Foundation under Award 0916887 and Award 1028807.

H. Liu and S. Datta are with the Department of Electrical Engineering, The Pennsylvania State University, University Park, PA 16802 USA (e-mail: hx1249@psu.edu; sdatta@engr.psu.edu).

M. Cotter and V. Narayanan are with the Department of Computer Science and Engineering, Pennsylvania State University, University Park, PA 16802 USA (e-mail: mjc324@cse.psu.edu; vijay@cse.psu.edu).

Color versions of one or more of the figures in this paper are available online at http://ieeexplore.iee.org.

Digital Object Identifier 10.1109/TDMR.2014.2316505

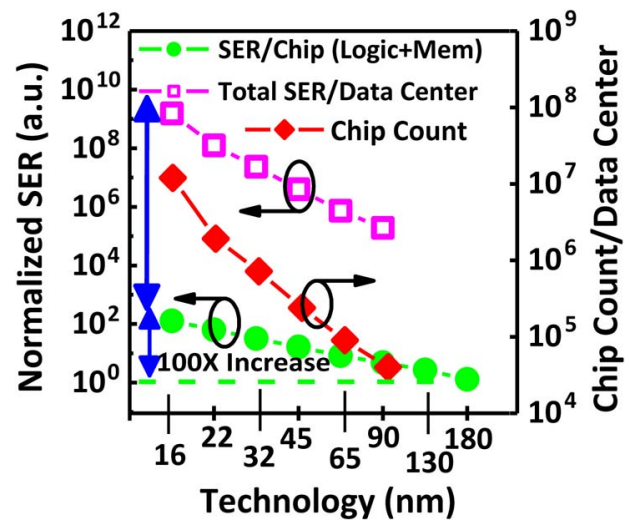

Fig. 1. SER per chip and total SER trend per data center with technology scaling and chip count increase [1], [2].

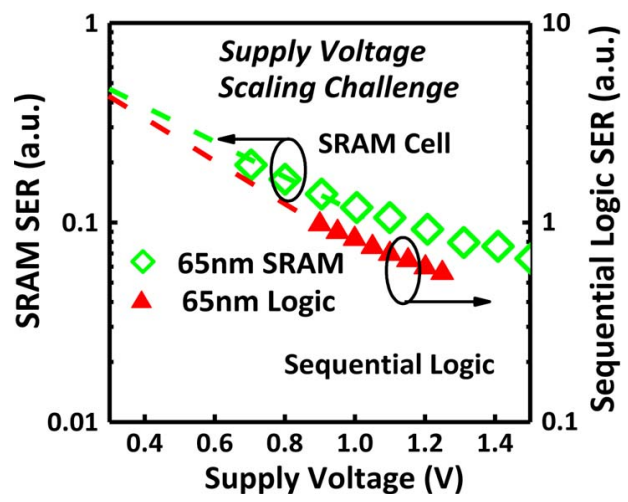

Fig. 2. Soft error rate (SER) with voltage scaling for $65 \mathrm{~nm}$ technology [4].

as the subthreshold circuit design has been widely studied to achieve the optimal energy efficiency with the tradeoff between overall throughput and performance at reduced supply voltages [3]. However, the reduction of the circuit node charge, which is proportional to the supply voltage, will induce significant increase of SER [4] as shown in Fig. 2, which may further prevent the projected voltage scaling [5] due to the reliability requirements.

To extend the improvement of energy efficiency along the technology roadmap, low bandgap materials (e.g., germanium, III-Vs) have been proposed as channel replacement for mobility enhancement [6]. The steep switching devices (e.g., III-V Tunnel TFET [7]-[9]) have also been demonstrated to overcome the non-scalable threshold voltage limit (tradeoff between offstate leakage and on-state drive strength) in CMOS technology. These emerging devices have offered new perspectives with improved performance and energy efficiency, especially 

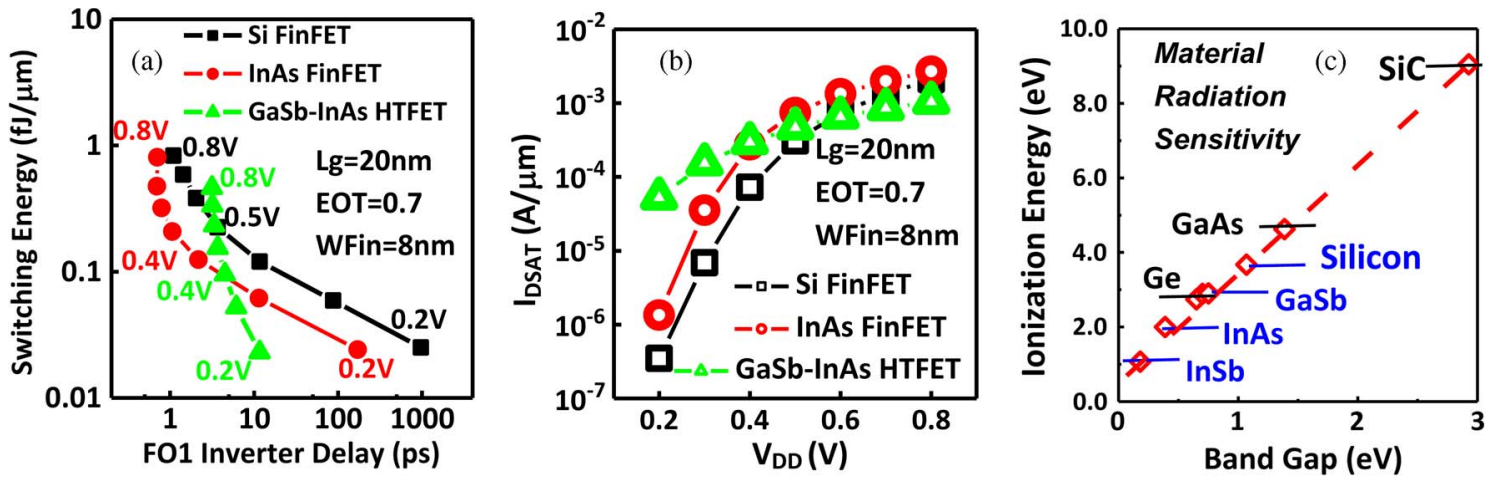

Fig. 3. (a) Switching energy vs. delay performance and (b) drive current IDSAT vs. supply voltage VDD for Si FinFET, III-V FinFET and HTFET. HTFET shows superior energy efficiency below $0.4 \mathrm{~V}$. (c) Radiation ionization energy with band gap for various semiconductor materials [12].

at reduced supply voltages [10], [11] as shown in Fig. 3(a) and (b). From the soft-error point of view, however, the narrow bandgap materials generally have low ionization energies [12], [13], which are more sensitive to neutron radiation compared to $\mathrm{Si}$ (Fig. 3(c)). Experimental and simulation studies on radiationinduced transient current generation in III-V HEMTs and HBTs have been performed in [14], [15], showing transient current generation difference due to material and device architecture change. However, the neutron induced charge generation analysis as well as the circuit-level SER has not been systematically investigated for low power emerging devices such as III-V FinFETs and TFETs.

In this paper, the soft-error generation and propagation in $\mathrm{Si}$ FinFET, III-V FinFET and III-V Hetero-junction Tunnel FET (HTFET) are investigated for technology assessment from a soft-error perspective. Due to the material and device architecture differences and lack of SPICE models for circuit analysis, we has studied the transient error generation using the device simulation and Verilog-A modeling technique [10] of these emerging devices. To evaluate the SER performance, we have developed the methodology based on the device simulation and critical LET extraction for SRAM and logic, and compared the SRAM bit-flip, electrical and latching window masking effects with supply voltage scaling.

The current paper is organized as follows. Section II discusses the charge collection and device-level transient current generation in Si FinFET, III-V FinFET and HTFET. Section III describes the SER evaluation methodology using device to circuit simulation. The critical LET extraction for SRAM and combinational logic as well as the SRAM bit-flip, electrical masking and latching window masking effects are presented in Section IV. Section V compares the SRAM SER and logic SER with voltage scaling for these three types of emerging devices, respectively. The conclusion is given in Section VI.

\section{TRANSIENT ERror Generation IN EMERGING DEVICES}

\section{A. Device Simulation and Baseline Setup}

In order to perform the radiation analysis using our highlyscaled and calibrated TCAD device models, we adopt the TCAD Sentaurus heavy ion model [17] to perform the radiation-induced transient current evaluation with the energy dependent impact ionization and hydrodynamic transport models. In our model, the generated electron/hole $(\mathrm{e} / \mathrm{h})$ pairs along the ion track are assumed to follow the Gaussian distribution with a spatial radius of $10 \mathrm{~nm}$, a characteristic time of $2 \mathrm{ps}$ and time distribution centered on 20 ps [18]. Linear energy transfer (LET) describes the charge deposition per length along the ion track, which is modeled as a fixed value along the track for fully depleted fin structure [19]. The ion strike is centered to the fin at normal incidence. The generated charge results in a transient current at the device off-state $\left(\mathrm{V}_{\mathrm{GS}}=0 \mathrm{~V}, \mathrm{~V}_{\mathrm{DS}}=\mathrm{V}_{\mathrm{DD}}\right)$.

Radiation-induced ion profiles have been studied through Monte Carlo simulation showing radial expansion of e/h pairs in micrometer range for $45 \mathrm{~nm}$ and beyond SOI technology with good agreement with the experimental data [16]. An underestimation of the radial expansion in long-channel devices may overestimate the soft-error rate due to the charge deposition confinement. In our work, the device models used for softerror performance analysis in the later discussion are based on ultra-thin body (UTB), double-gate structures with $20 \mathrm{~nm}$ channel length. The ion induced charge deposition is assumed in the channel. The UTB structure is due to the electrostatic control requirement in TFET design to outperform Si FinFET at $20 \mathrm{~nm}$ and beyond. Here, it can effectively reduce the device terminal junction area and the length of the ion tracks in vertical direction, constraining the charge deposition in the top device layer. For the lateral expansion of the ion profile, because the device integration of these advanced emerging III-V technologies with specific engineering of the material layers and new device architectures (e.g., HTFET) requires additional device isolations, which can reduce the charge deposition effect outside the gate region. Thus, the choice of $10 \mathrm{~nm}$ radial charge expansion in our work is corresponding to the worst case scenario. Further study on the device source/drain design, layout and surrounding structure will improve our models for practical applications.

A study of the charge collection with fin width scaling is performed to set-up a baseline structure on three-dimensional $\mathrm{Si}$ FinFET with $20 \mathrm{~nm}$ gate length $\left(\mathrm{L}_{\mathrm{g}}\right), 25 \mathrm{~nm}$ fin height $\left(\mathrm{H}_{\text {Fin }}\right)$ and $0.7 \mathrm{~nm}$ EOT (Fig. 4). The charge collection at the drain node at $\mathrm{V}_{\mathrm{DD}}=0.5 \mathrm{~V}$ and $0.8 \mathrm{~V}$ with LET $=50 \mathrm{fC} / \mu \mathrm{m}$ is shown in Fig. 5. Due to the reduction of the bulk collection [19], the total collected charge $\left(Q_{\text {coll }}\right)$ at $1 \mathrm{~ns}$ after ion strike and the collected charge to the node charge ratio $\left(\mathrm{Q}_{\text {coll }} / \mathrm{Q}_{\text {node }}\right)$ 


\section{FinFET Structure}

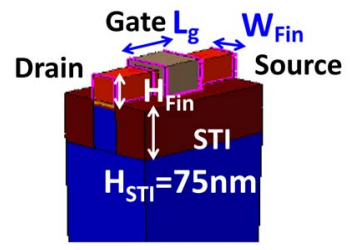

lon Strike

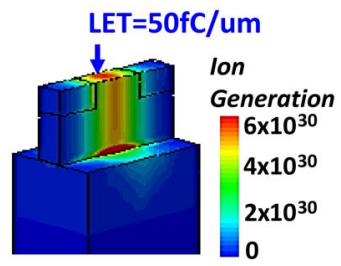

Fin Width Reduction:

Sensitive Area and Charge Collection Reduction

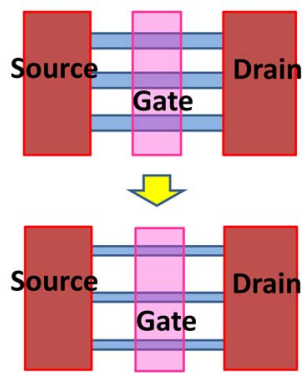

Fig. 4. Sensitive area reduction and charge collection reduction with fin width scaling due to bulk collection reduction.
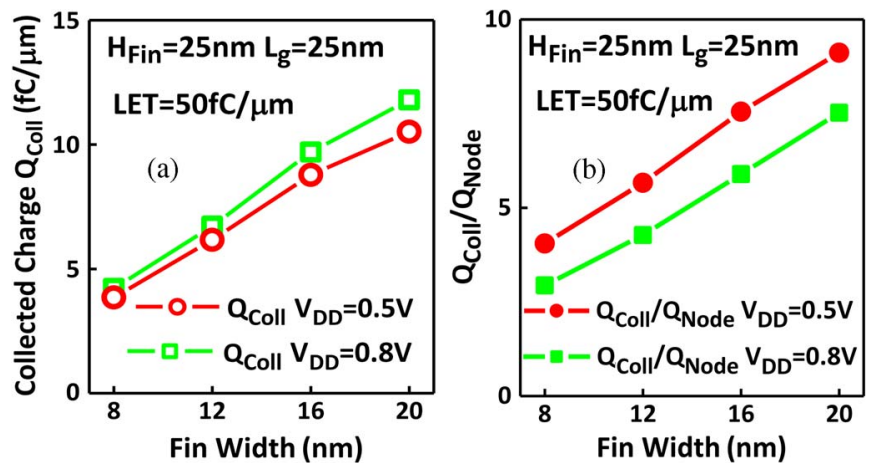

Fig. 5. (a) Charge collection with fin width (b) collected charge to node charge ratio with fin width at $\mathrm{V}_{\mathrm{DD}}=0.5 \mathrm{~V}$ and $0.8 \mathrm{~V}$.

TABLE I

Device Simulation Parameter Setup

\begin{tabular}{|l|c|}
\hline Gate Length $\left(\mathrm{Lg}_{\mathrm{g}}\right)$ & $20 \mathrm{~nm}$ \\
\hline EOT $\left(\mathrm{HfO}_{2}\right)$ & 0.7 \\
\hline Fin Width $\left(\mathrm{t}_{\text {Body }}\right)$ & $8 \mathrm{~nm}$ \\
\hline InAs FinFET Source/Drain Doping & $4 \times 10^{19} \mathrm{~cm}^{-3}$ \\
\hline Si FinFET Source/Drain Doping & $1 \times 10^{20} \mathrm{~cm}^{-3}$ \\
\hline HTFET Source $(\mathrm{GaSb})$ Doping & $4 \times 10^{19} \mathrm{~cm}^{-3}$ \\
\hline HTFET Drain $(\mathrm{InAs})$ Doping & $2 \times 10^{17} \mathrm{~cm}^{-3}$ \\
\hline
\end{tabular}

both decrease with the fin width $\left(\mathrm{W}_{\text {Fin }}\right)$ scaling from $20 \mathrm{~nm}$ to $8 \mathrm{~nm}$. $W_{\text {Fin }}$ reduction can also effectively reduce the radiation sensitive area [20], which further improves the overall radiation resilience.

In the following simulation, a fin width of $8 \mathrm{~nm}$ (UTB) is used as baseline in a double-gate structure with $20 \mathrm{~nm}$ gate length for Si FinFET, InAs FinFET and GaSb-InAs HTFET. With the quantum confinement effect, GaSb bandgap $\mathrm{E}_{\mathrm{g}, \mathrm{GaSb}}=0.804 \mathrm{eV}$, InAs bandgap $\mathrm{E}_{\mathrm{g}, \text { InAs }}=0.44 \mathrm{eV}$ and a conduction band offset $\Delta \mathrm{E}_{\mathrm{c}}=0.796 \mathrm{eV}$ for hetero-interface are used in HTFET simulation. Table I shows the key parameters in the device simulation. The DC characteristics for Si and III-V FinFETs, which are shown in Fig. 6, have been calibrated with experimental data [21]. The HTFET model has been calibrated in [10] with atomistic non-equilibrium Green's function formalism (NEGF) simulation (a ballistic, full band, two-

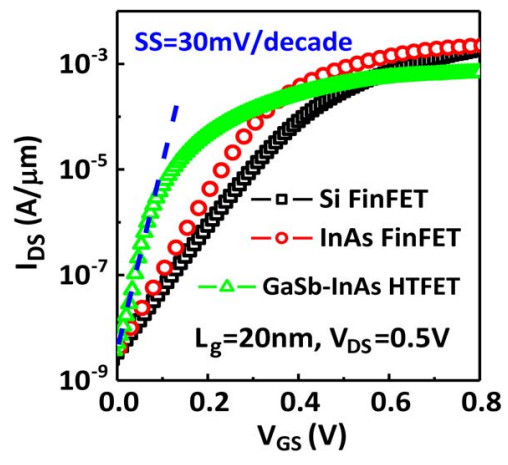

Fig. 6. $\mathrm{DC}\left(\mathrm{I}_{\mathrm{ds}}-\mathrm{V}_{\mathrm{gs}}\right)$ characterization and simulation parameters (Table I) of Si FinFET, InAs FinFET and GaSb-InAs HTFET at $\mathrm{V}_{\mathrm{DS}}=0.5 \mathrm{~V}$. Average subthreshold slope (SS) of $30 \mathrm{mV} /$ decade over 2 orders of $\mathrm{I}_{\mathrm{DS}}$ change is achieved in HTFET.

and three-dimensional Schrodinger-Poisson solver to study the quantum transport in various semiconductor devices [22] as a precise simulation approach). At $\mathrm{V}_{\mathrm{DS}}=0.5 \mathrm{~V}$, HTFET shows improved $\mathrm{I}_{\mathrm{DS}}$ below $\mathrm{V}_{\mathrm{GS}}=0.5 \mathrm{~V}$ and $\mathrm{V}_{\mathrm{GS}}=0.3 \mathrm{~V}$ compared to Si FinFET and InAs FinFET, respectively. The average subthreshold slope (SS) is $30 \mathrm{mV} / \mathrm{dec}$, which is extracted over 2 decades of current change.

\section{B. Transient Current Generation}

In fully-depleted-channel devices (e.g., SOI MOSFETs, FinFETs), the transient current generation and charge collection are significantly affected by the bipolar gain effect [23], [24]. In nMOSFETs, the generated electrons in the channel are collected at the drain node due to the source-drain bias. The generated holes, on the other hand, are stored in the body due to the source-channel barrier, increasing the channel potential. The additional electrons flow into the channel and further increase the drain node charge collection. The ratio of the total collected charge, $\mathrm{Q}_{\text {coll }}$, at the drain node to the deposited charge, $\mathrm{Q}_{\mathrm{dep}}$, is known as bipolar effect coefficient.

The time evolutions of the hole density in the channel for nMOSFETs and nHTFETs is shown in Fig. 7. The ion strikes the channel center at $t=20 \mathrm{ps}$ for each device. The higher initial hole concentration in III-V FinFET at $t=0$ ps before the ion strike is due to the reduced density of states in InAs compared to Si. For both Si FinFET and III-V FinFET, the hole storage in the channel is clearly observed after the ion strike as discussed, causing the bipolar gain effect and additional charge collection. For nHTFET, however, the hole density in the channel region decreases fast. Similar response is also observed for the electron density in pHTFET as shown in Fig. 8.

The suppression of the hole storage in HTFET can be explained from the HTFET device operation. HTFET is essentially a reverse biased $\mathrm{p}-\mathrm{i}-\mathrm{n}$ diode with asymmetric source/drain doping. The deposited electrons can be collected at drain node $(\mathrm{n}+$ region) similar as in MOSFETs, while the holes can be collected at the source node $(\mathrm{p}+$ region) at the same time, rather than being stored in the channel as in MOSFETs. This charge collection through the ambipolar transport leads to significant hole storage reduction in HTFET. Thus, the reduction of the minority carrier storage (e.g., hole storage in n- HTFET) in 
Hole Density in n-type Device Channel Region

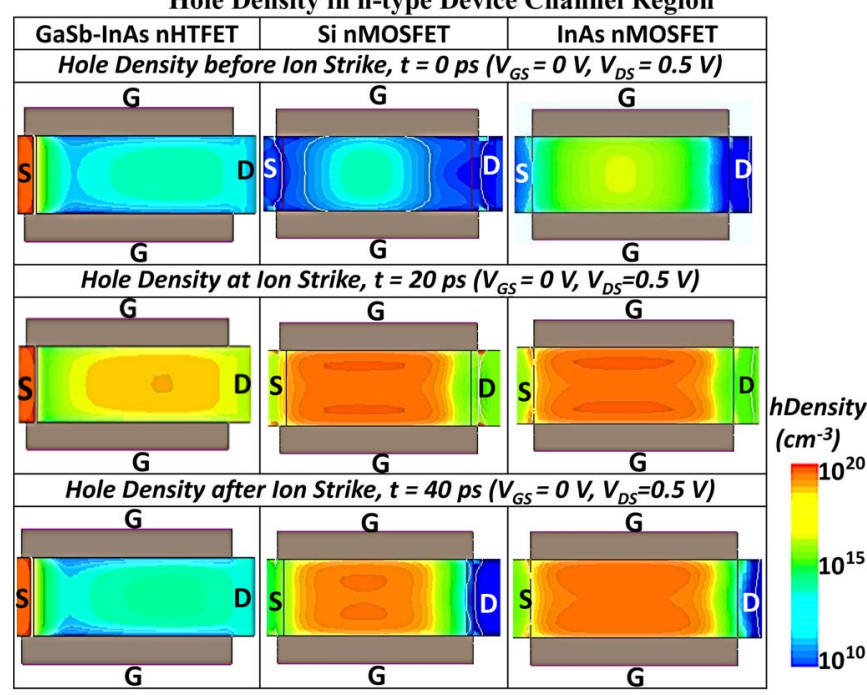

Fig. 7. Time evolution of hole density in n-type device channel region. Hole density decreases quickly in nHTFET due to ambipolar transport. Hole storage due to radiation induced charge deposition is observed in $\mathrm{Si}$ and $\mathrm{InAs}$ nMOSFETs, which induces the bipolar gain effect.

Electron Density in p-type HTFET Channel Region

\begin{tabular}{|c|c|c|c|}
\hline$t=0$ ps, Before Strike & $t=20 \mathrm{ps}$, lon Strike & $t=40 \mathrm{ps}$, After Strike & eDensity \\
\hline G & G & G & $\left(\mathrm{cm}^{-3}\right)$ \\
\hline D & D & D & $10^{13}$ \\
\hline G & G & G & 1 \\
\hline
\end{tabular}

Fig. 8. Time evolution of electron density in p-type HTFET. Electron density decreases quickly in HTFET.

the channel can significantly reduce the bipolar gain effect and additional charge injection $\left(Q_{\text {inj }}\right)$ in HTFET.

To analyze the contributions of different factors that impact transient current generation and charge collection, we further evaluated the charge collection $\left(\mathrm{Q}_{\text {coll }}\right)$ and charge recombination $\left(\mathrm{Q}_{\mathrm{rec}}\right)$, given the charge conservation $Q_{\text {coll }}=Q_{\text {inj }}-Q_{\text {rec }}$. Fig. 9 shows the band diagram before and after the ion strike for nMOSFETs and nHTFET, which reveals the hole storage induced source barrier lowering effect. In Si FinFET, the hole storage causes $0.119 \mathrm{eV}$ source barrier reduction $(10 \%$ of $\mathrm{Si}$ bandgap, $\mathrm{E}_{\mathrm{g}, \mathrm{Si}}$ ) at a distance of $2 \mathrm{~nm}$ from the source-channel junction toward the channel region, as compared to $0.0044 \mathrm{eV}$ $\left(1 \% \mathrm{E}_{\mathrm{g}, \mathrm{InAs}}\right)$ reduction in HTFET. Due to the thermionic emission induced carrier injection in MOSFETs, this significant source barrier reduction results in an exponential increase of current (as device turns on), which further increases $Q_{i n j}$ in $\mathrm{Si}$ and III-V FinFETs. Moreover, we evaluated the charge recombination in the channel region as shown in Fig. 10(a) and (b) at $1 \mathrm{~ns}$ after the ion-strike. HTFET and III-V FinFET show higher Shockley-Read-Hall (SRH) recombination rate due to InAs-channel (reduced bandgap and carrier lifetime) compared to Si FinFET (Fig. 10(a)) [17], while Si and III-V FinFETs exhibit higher Auger recombination due to larger $Q_{\text {inj }}$ [17] from the source barrier lowering (Fig. 10(b)). HTFET and III-V FinFET show overall higher $Q_{\text {rec }}$ (including both SRH and Auger recombination) than Si FinFET.

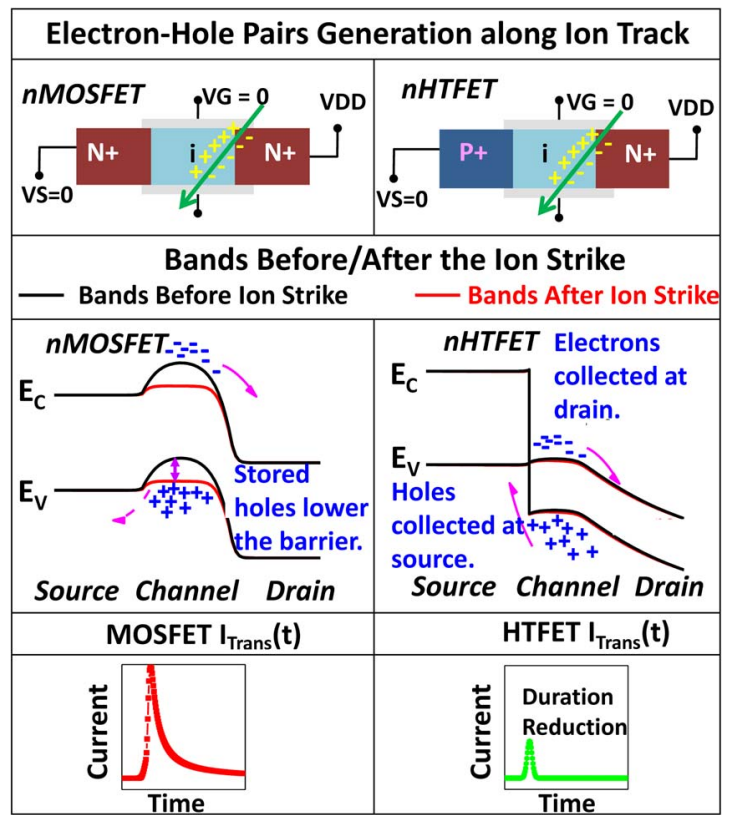

Fig. 9. Band diagrams for nMOSFET and nHTFET before/after ion strike. Hole storage induces barrier lowering and additional charge collection (bipolar gain) in nMOSFET. For nHTFET, holes can be collected at source, which reduces the bipolar gain effect.
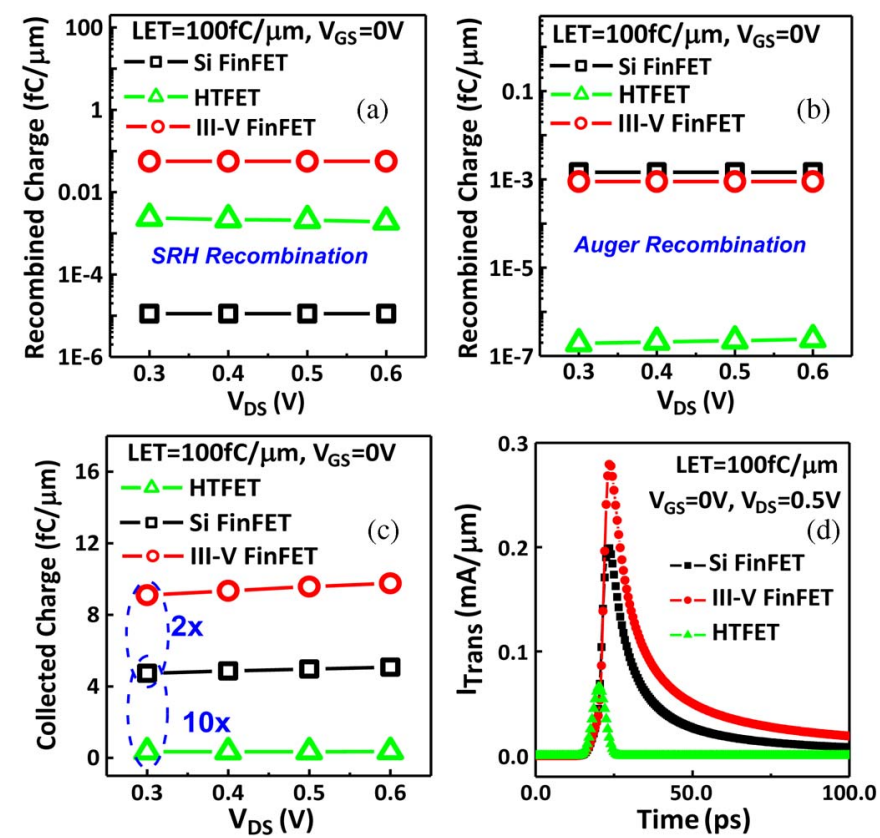

Fig. 10. Radiation induced charge recombination through (a) SRH recombination and (b) Auger recombination, (c) charge collection at $1 \mathrm{~ns}$, and (d) transient current profile for each emerging device (LET $=0.1 \mathrm{pC} / \mu \mathrm{m}$ ). HTFET shows reduced current magnitude and 10x charge collection reduction compared to Si FinFET. 2x charge collection enhancement is observed in III-V FinFET compared to Si FinFET due to high carrier mobility.

The depletion region volume and the electric field also strongly affect the charge collection process and drift current [28], [30]. Compared to the large reverse bias that exists between the channel and the drain in MOSFETs, HTFET exhibits a very small potential drop between the channel and drain due to its $\mathrm{p}-\mathrm{i}-\mathrm{n}$ structure and the reduced drain doping (to suppress the ambipolar operation) [32], which is also consistent with the 


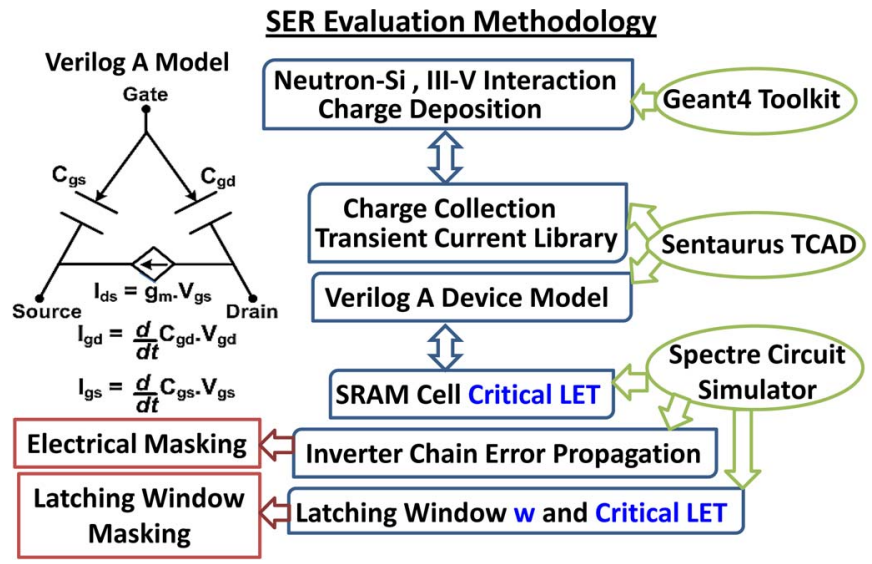

Fig. 11. SER evaluation methodology.

band bending (Fig. 9). 90\% of electric field reduction can be achieved in the depleted channel of HTFET as compared to $\mathrm{Si}$ FinFET at $\mathrm{V}_{\mathrm{ds}}=0.5 \mathrm{~V}$, which reduces the charge separation and the drift current magnitude.

Fig. 10(c) and (d) show the resulted $Q_{\text {coll }}$ at $1 \mathrm{~ns}$ after the ion strike at $\mathrm{LET}=100 \mathrm{fC} / \mathrm{um}$ for different $\mathrm{V}_{\mathrm{DD}}$ and transient current profile at $\mathrm{V}_{\mathrm{DD}}=0.5 \mathrm{~V}$ comparing Si FinFET, III-V FinFET and HTFET. Q coll of approximately 6 times and 10 times over the initial deposited charge are observed for $\mathrm{Si}$ and InAs FinFETs, respectively, while $60 \%$ of the deposited charge is collected in HTFET. Compared to Si FinFET, a vast majority $(90 \%)$ of the $Q_{i n j}$ reduction is due to suppression of the source barrier lowering and the bipolar gain effect. This is the dominant factor for much reduced $\mathrm{Q}_{\text {coll }}$ in HTFET, considering $Q_{\text {rec }}$ is relatively small compared to $\mathrm{Q}_{\text {coll }}$. The increased $\mathrm{Q}_{\text {rec }}$ (1.4x compared to Si FinFET) and the reduction of electric field in HTFET further reduces $\mathrm{Q}_{\text {coll }}$ and drift current (70\% reduction of magnitude). Overall, HTFET exhibits approximately $80 \%$ reduction in transient duration compared to Si FinFET, while III-V FinFET shows higher transient current magnitude compared to Si FinFET due to the high channel carrier mobility, which results in enhanced charge collection.

\section{Soft-Error Evaluation Methodology}

The soft-error rate evaluation methodology used in this paper is shown in Fig. 11, which includes the material-level charge deposition evaluation, device-level charge collection evaluation and transient current profile generation as well as circuit-level critical LET extraction. The sea-level neutron induced charge deposition in materials such as InAs (channel material for III-V FinFET and HTFET) and Si is obtained from Geant4 [25] Monte Carlo simulation using the measured neutron spectrum [26]. The charge deposition in terms of LET is then applied to the TCAD device simulation to obtain the transient current characteristics for each type of nanoscale device at different $\mathrm{V}_{\mathrm{DD}}$ and radiation strength as discussed in the previous section, which is later on applied as input to the strike node in the circuit analysis. For circuit simulation, a lookup table based Verilog-A models generated from TCAD Sentaurus has been used for each type of device based circuit implementation and
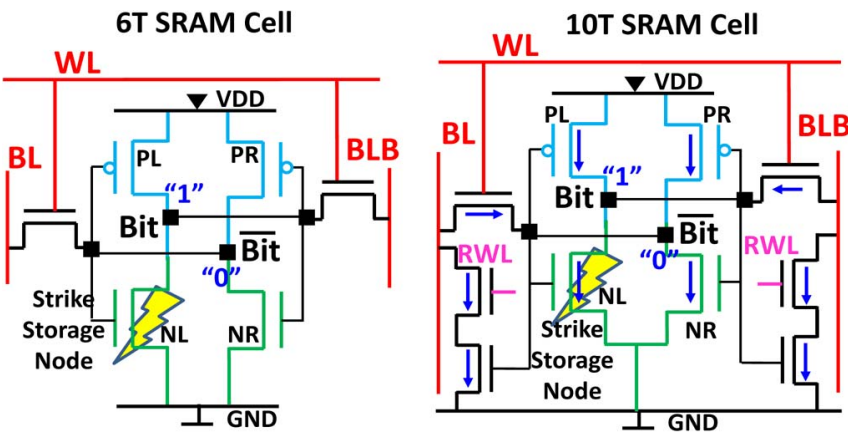

Fig. 12. 6T and 10T SRAM cell schematic. Strike on storage node induced charge exceeding node charge can cause an error. HTFET (unidirectional) current flow direction is illustrated for 10T cell.

soft-error analysis using Spectre [27]. The circuits evaluated in this work include 6T and 10T SRAM cells, FO1 inverter chain and NAND gate based D Flip-flop to study the SRAM bit-flip, combinational logic electrical and latching window masking effects. The critical LETs for SRAM cell and combinational logic are extracted with voltage scaling for SER calculation. Technology adaptable empirical model [28], which is validated on previous CMOS technology nodes, is applied to SER calculation for each emerging device based circuits

$$
\begin{aligned}
& S E R_{S R A M}\left(L E T_{\text {critical }}\right) \sim A \cdot\langle\text { Flux }\rangle \\
& \quad \cdot \exp \left(-Q_{\text {critical }} /\left\langle Q_{s}\right\rangle\right) \sim \exp \left(-L E T_{\text {critical }} /\left\langle L E T_{s}\right\rangle\right) . \\
& S E R_{\text {Logic }}\left(L E T_{\text {critical }}\right) \sim w / c \\
& \quad \cdot \exp \left(-L E T_{\text {critical }} /\left\langle L E T_{s}\right\rangle\right) . \\
& Q_{\text {critical }}=t_{\text {body }} \cdot L E T_{\text {critical }} .
\end{aligned}
$$

Here, $S E R_{S R A M}$ and $S E R_{\text {Logic }}$ represent SRAM cell SER and combinational logic SER, respectively. $A$ refers to the radiation sensitive device area, $t_{\text {body }}$ is the double-gate structure body thickness, and $\langle F l u x\rangle$ is the average neutron flux over the energy spectrum at the sea level. $Q_{\text {critical }}$ is the SRAM cell bit-flip critical charge, and $\left\langle Q_{s}\right\rangle$ is the average charge collection coefficient in the exponential function, both of which can be converted to $L E T_{\text {critical }}$ and $\left\langle L E T_{s}\right\rangle$ using (3) [23]. $S E R_{\text {Logic }}$ is proportional to error latch probability $w / c$ [30], where $w$ is the latching window and $c$ is the clock cycle. Typical $\left\langle L E T_{s}\right\rangle$ for $\mathrm{Si}$ is $50 \mathrm{fC} / \mu \mathrm{m}$ at sea-level [29], $\left\langle L E T_{s}\right\rangle$ for InAs is obtained from Geant4 simulation.

\section{CRitical LET Evaluation in Circuits}

\section{A. SRAM Critical LET Evaluation}

Softs errors occur in SRAM cells when the ion strike induced collected charge exceeds the storage node charge causing the bit-flip. Fig. 12 shows the 6T and 10T SRAM cell schematics with neutron strike at the nMOSFET or nTFET connected to the bit node, which stores "1". 10T SRAM cell here uses iso-area sizing according to the reported TFET SRAM design in [10] to compare with 6T. The SRAM Critical LET is extracted at the threshold LET causing the bit-flip [29]. The 6T and iso-area 10T SRAM cell critical LET extraction is performed for both $\mathrm{Si}$ FinFET and III-V FinFET, while only the 10T SRAM critical 

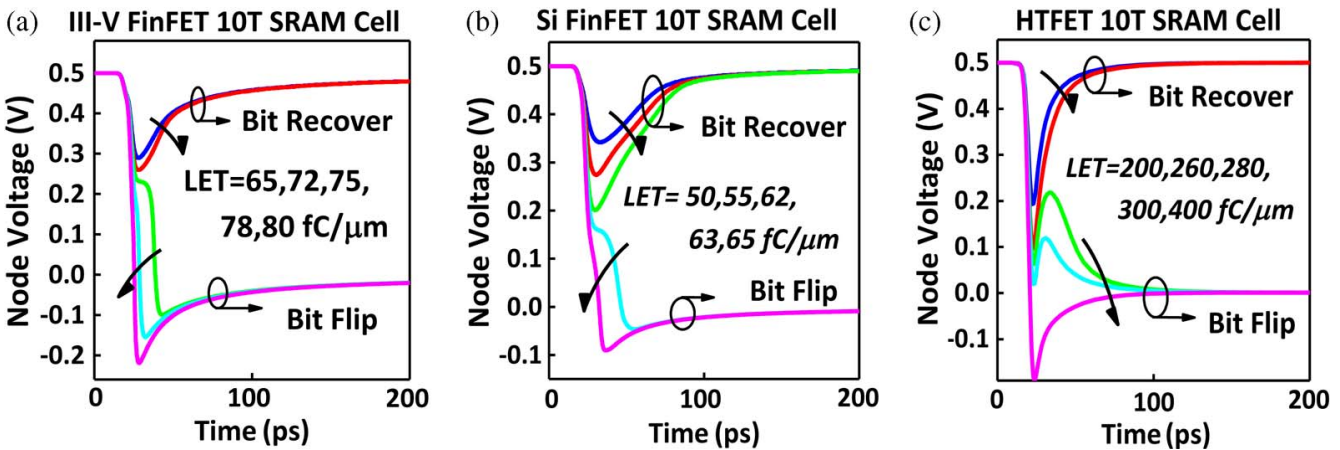

Fig. 13. 10T SRAM cell critical LET extraction and node bit-flip illustration for (a) Si FinFET, (b) III-V FinFET, and (c) HTFET at V VD $=0.5$ V. HTFET shows high critical LET and short recovery time.
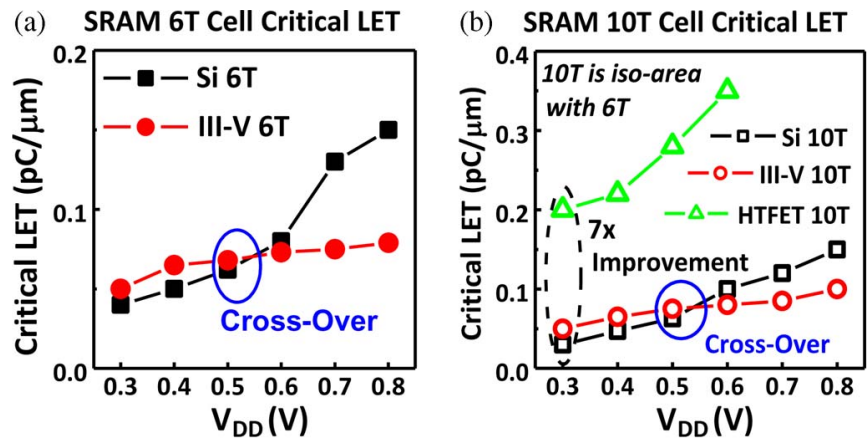

Fig. 14. (a) $6 \mathrm{~T}$ and (b) 10T iso-area SRAM cell critical LET extraction for Si FinFET, III-V FinFET and 10T HTFET with voltage scaling. HTFET shows 7 times improvement compared to Si FinFET at $0.3 \mathrm{~V}$ for $10 \mathrm{~T}$ case.

LET extraction is performed for HTFET. This is because of TFET's asymmetrical source/drain and uni-directional current flow characteristic, which requires additional current paths for stable read and write. It has been reported in [10] the 10T HTFET cell can achieve a desired noise margin compared to MOSFET designs.

The bit-flip comparison with LET increase is shown in Fig. 13(a)-(c) for Si, III-V FinFET and HTFET 10T SRAM cell at $0.5 \mathrm{~V}$. HTFET shows improved critical LET and reduced recovery time (transient duration from the strike time to $90 \%$ node voltage recovery). The extracted critical LET for 6T (except HTFET) and iso-area10T SRAM cell is plotted with voltage scaling as shown in Fig. 14. For all the evaluated cells, the critical LET decreases with the supply voltage due to the reduced charge of the storage node. Above $0.5 \mathrm{~V}$, Si FinFET cell shows higher critical LET compared to III-V FinFET, which is due to the reduced charge collection. Below $0.5 \mathrm{~V}$, however, the critical LET for Si FinFET cell decreases significantly due to the low drive current near threshold operation, where III-V FinFET with improved drive current shows the cross-over. Compared to Si FinFET, HTFET shows 4.5 times improvement at $\mathrm{V}_{\mathrm{DD}}=0.5 \mathrm{~V}$ and 7 times improvement at $\mathrm{V}_{\mathrm{DD}}=0.3 \mathrm{~V}$ of the critical LET, and $50 \%$ recovery time reduction. This is due to the short transient current duration and the reduced charge collection (discussed in the Section II-B), as well as the enhanced on-state Miller capacitance effect [32].

Due to the asymmetrical p-i-n structure and tunneling mechanism, TFET exhibits different capacitance characteristics compared to MOSFET. In MOSFET, both the gate-to-source
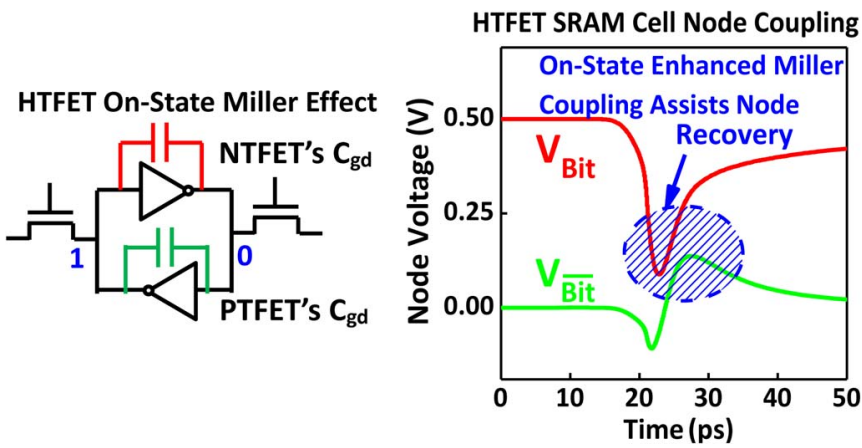

Fig. 15. HTFET on-state enhanced Miller capacitance illustration in SRAM cell and node recovery process with bit and $\overline{b i t}$ node coupling.
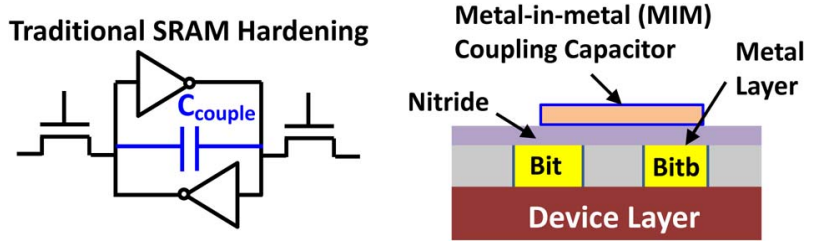

Fig. 16. Metal-in-metal coupling capacitor in SRAM cell and cross-section schematics for traditional CMOS SRAM hardening.

capacitance, $\mathrm{C}_{\mathrm{gs}}$, mainly contribute to the total gate capacitance, $\mathrm{C}_{\mathrm{gg}}$ at the device on-state. In HTFET, however, the gateto-drain capacitance, $\mathrm{C}_{\mathrm{gd}}$, is dominant in $\mathrm{C}_{\mathrm{gg}}$, while $\mathrm{C}_{\mathrm{gs}}$ is suppressed at on-state. This enhanced on-state $\mathrm{C}_{\mathrm{gd}}$ in HTFET can increase the input (gate) to output (drain) coupling during switching, which is known as enhanced on-state Miller capacitance [32]. For soft-error performance, the enhanced on-state Miller capacitance in HTFET assists the node recovery process. As shown in Fig. 15, the feedback from the HTFET bit node to bit node can improve the critical LET, which is similar to the metal-insulator-metal (MIM) coupling capacitance (Fig. 16) employed for traditional backend SRAM radiation hardening [33]. For the evaluated voltage range of $0.3 \mathrm{~V}$ to $0.6 \mathrm{~V}$, HTFET presents superior soft-error resilience compared to Si FinFET and III-V FinFET.

\section{B. Combinational Logic Critical LET Evaluation}

In the combinational logic, the generated transient current induces a voltage pulse at circuit node, which is propagated through the circuit path. Due to the delay of the logic gates [34], 
(a) Electrical Masking

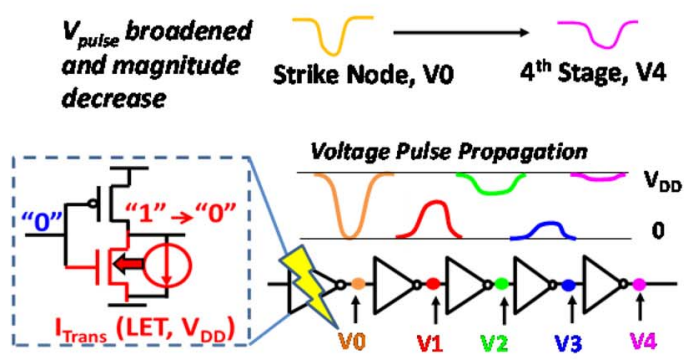

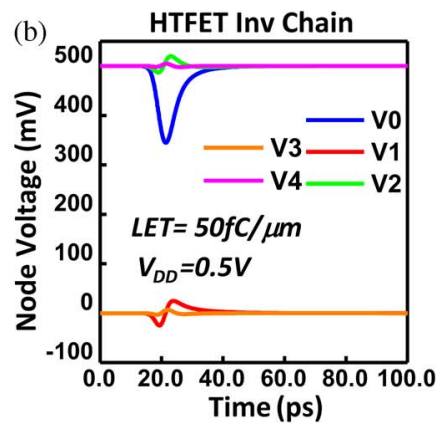

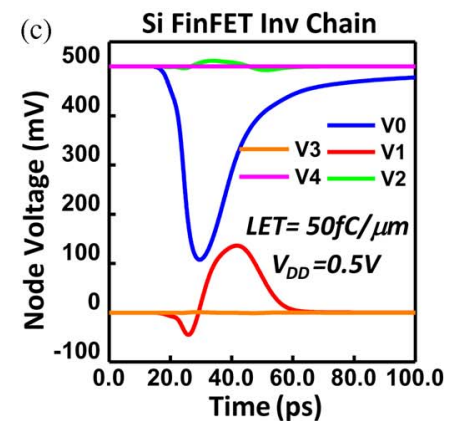

Fig. 17. (a) Electrical masking effect illustration using FO1 inverter chain. The strike is induced at the 1st stage nFET, where the transient current causing a voltage pulse at node V0. (b) Transient voltage propagation at each node from $\mathrm{V}_{0}$ to $\mathrm{V}_{4}$ for $\mathrm{HTFET}$ and (c) $\mathrm{Si}$ FinFET FO1 inverter chain at LET $=50 \mathrm{fC} / \mu \mathrm{m}$ strike.
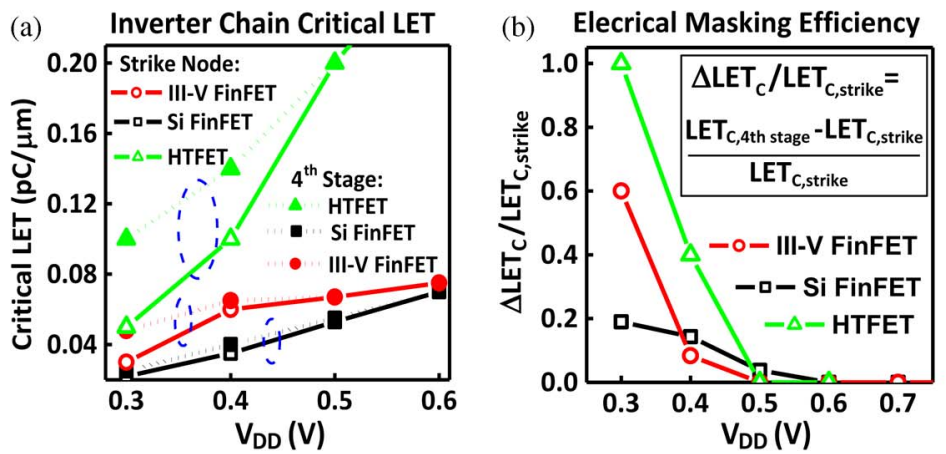

(c) Latching Window Masking

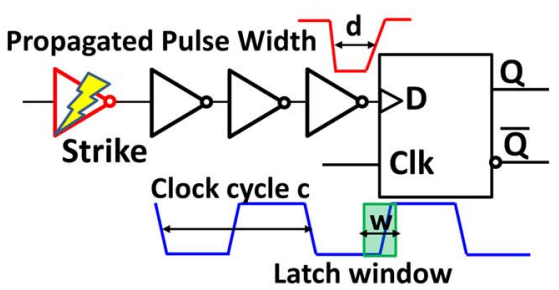

A pulse with $d>w$ can be latched. Latching Probability: $w / c$

Fig. 18. (a) Inverter chain critical LET (causing $\mathrm{V}_{\text {pulse }}>0.5 \mathrm{~V}_{\mathrm{DD}}$ ) at the strike node and the 4th inverter stage and (b) normalized critical LET difference between strike node and 4th stage with voltage scaling. (c) DFF latch window masking schematic. Propagated transient voltage pulse width $d$ exceeding the latch window $w$ can be latched with the probability of $w / c$.

the voltage pulse $\left(\mathrm{V}_{\text {pulse }}\right)$ can be masked with certain number of stages during propagation, which is known as the electrical masking effect. Fig. 17(a) illustrates the error propagation evaluation using FO1 inverter chain, showing the electrical masking effect. The strike induced transient current causes a voltage pulse at the strike node, which decreases during propagation. Fig. 17(b) and (c) compares the transient voltage propagation in HTFET and Si FinFET based FO1 inverter chains at $\mathrm{V}_{\mathrm{DD}}=$ $0.5 \mathrm{~V}$. HTFET shows faster voltage degradation and reduced transient voltage duration, which is consistent with transient current characteristics (Section II). The critical LET is extracted as the threshold LET which causes the propagated $V_{\text {pulse }}$ magnitude exceeding $0.5 \mathrm{~V} \mathrm{~V}$ DD.

Fig. 18(a) shows the critical LET with voltage scaling at the strike node and 4th stage, respectively. The increase of the critical LET between the strike node and the 4th stage in the inverter chain represents the masking effect. As shown in Fig. 18(b), the difference of critical LET between the strike and the 4th node is normalized to the critical LET of the strike node, showing the electrical masking efficiency. For all the evaluated devices, the inverter chain critical LET decreases with voltage scaling, while the electrical masking effect increases due to the increase of circuit delay with reduced $\mathrm{V}_{\mathrm{DD}}$. At low $\mathrm{V}_{\mathrm{DD}}$, the masking strength as well as the circuit radiation resilience is dominated by the drive current. As a result, below $0.4 \mathrm{~V}$, III-V FinFET shows improved electrical masking effect compared to Si FinFET due to the mobility enhancement. HTFET presents superior masking effect below $0.5 \mathrm{~V}$ due to the abrupt switching and improved current performance. Additionally, because of the reduced transient duration (reduce bipolar gain effect) and enhanced Miller capacitance, the critical LET for HTFET of all the evaluated voltage range is greatly improved compared to $\mathrm{Si}$ and III-V FinFET.

Error occurs in the state element requires: (1) the transient $\mathrm{V}_{\text {pulse }}$ width $d$ exceeds latch window $w$, which determines the latching window critical LET; (2) the transient error propagates to the state element during latching process. The error latched probability is then proportional to the latch window $w$ to the clock cycle $c$ ratio, $w / c$. The evaluation schematic of the latching window masking effect is shown in Fig. 18(c) using the NAND gate based D flip-flop (DFF). Fig. 19(a) shows the extracted $w$ of Si FinFET, III-V FinFET and HTFET DFFs with voltage scaling. Due to the steep switching, HTFET DFF shows reduced $w$ (improved circuit speed) at low $\mathrm{V}_{\mathrm{DD}}$, which can outperform Si and III-V FinFET DFF below $0.6 \mathrm{~V}$ and $0.4 \mathrm{~V}$, respectively. The propagated voltage pulse $d$ is then extracted with 4 stages of electrical masking. Fig. 19(b) shows the extracted $d$ at $\mathrm{V}_{\mathrm{DD}}=0.5 \mathrm{~V}$ with the lower LET electrically masked. The latching window critical LET is then extracted at $w=d$ at different $V_{\text {DD }}$. As shown in Fig. 19(c), HTFET shows overall improved latching window masking effect with improved critical LET compared to Si and III-V FinFET, and 8 times improvement of critical LET over Si FinFET is observed at $\mathrm{V}_{\mathrm{DD}}=0.3 \mathrm{~V}$. Above $0.5 \mathrm{~V}$, III-V FinFET shows lower critical LET compared to Si FinFET due to the reduced $w$ and enhanced charge collection. III-V FinFET outperforms Si FinFET due to the improved current drive below $0.5 \mathrm{~V}_{\mathrm{DD}}$. 

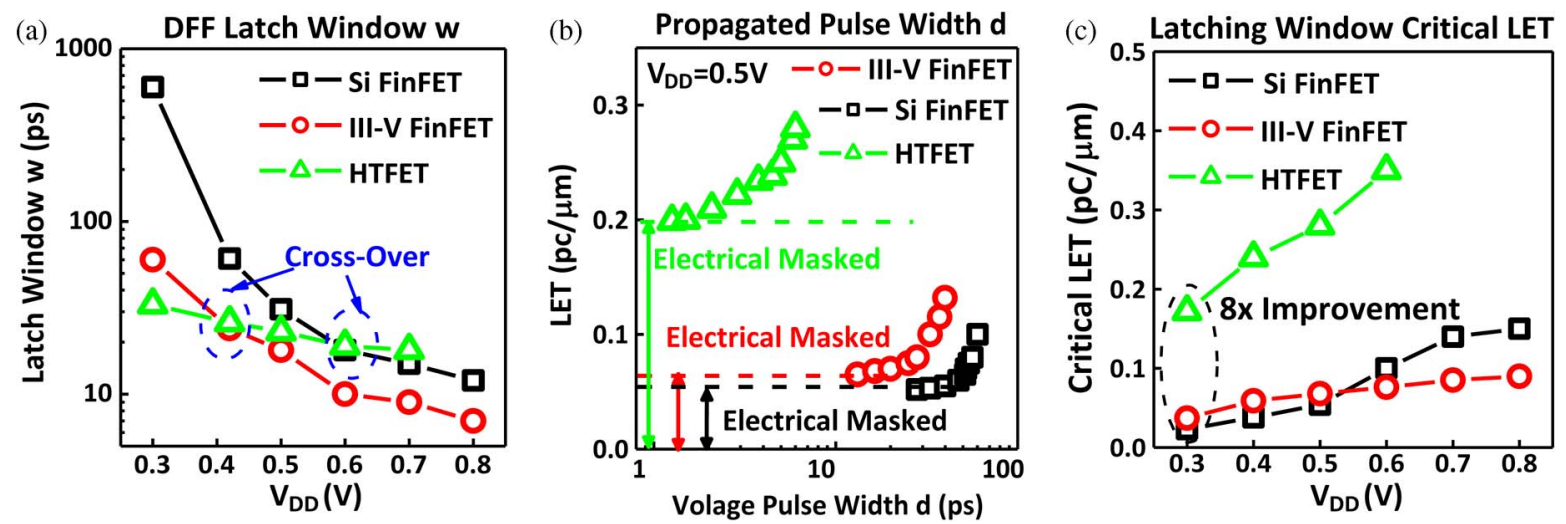

Fig. 19. (a) Latch window $w$ and (b) voltage pulse width $d$ characterization at $0.5 \mathrm{~V}$ for each device. HTFET shows reduced pulse width at the same radiation strength (LET) and higher threshold LET which can be electrical masked. (c) Latching window critical LET is extracted at $w=d$. HTFET shows 8 times critical LET improvement compared to Si FinFET at $0.3 \mathrm{~V}$. III-V FinFET shows cross-over at $0.5 \mathrm{~V}$ due to reduced $w / c$.
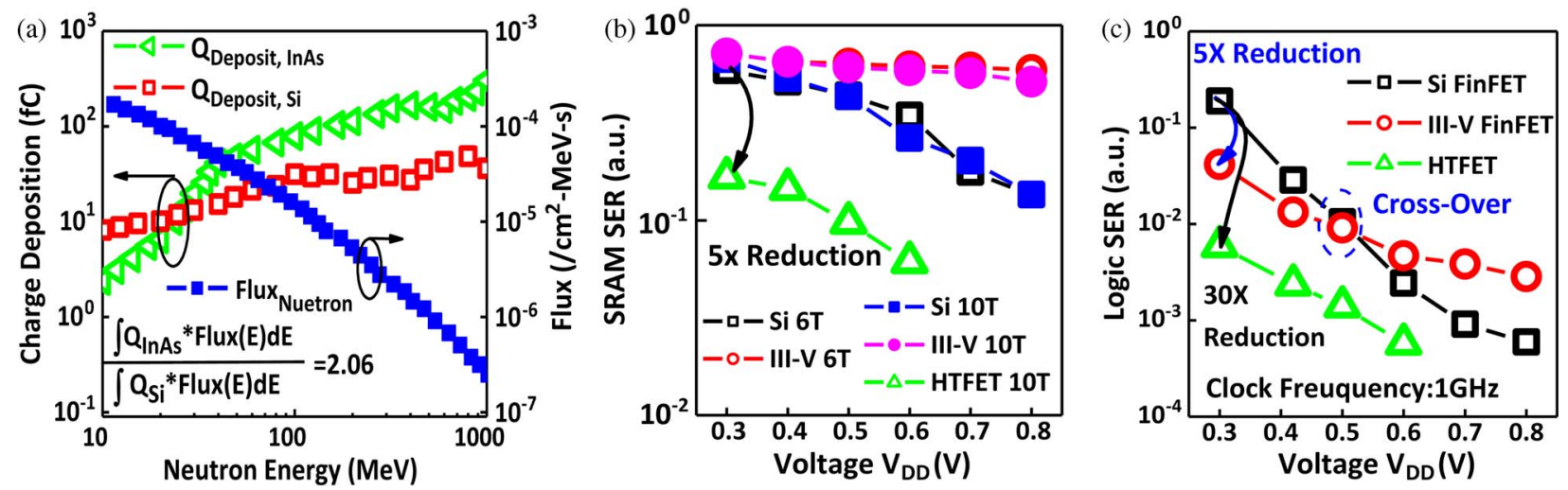

Fig. 20. (a) Monte Carlo simulation for neutron induced charge deposition on Si and InAs. InAs shows twice the charge deposition over the neutron spectrum [26] integration. (b) SRAM SER and (c) logic SER comparison with voltage scaling for Si, III-V FinFETs and HTFET. HTFET shows superior soft error resilience for both SRAM and logic. III-V FinFET logic shows lower SER below $0.5 \mathrm{~V}$ over Si FinFET logic.

\section{SER Evaluation}

\section{A. Neutron Induced Charge Deposition}

The neutron induced charge deposition is shown in Fig. 20(a) for InAs and Si. The evaluated sea-level neutron energy range is from $10 \mathrm{MeV}$ to $1000 \mathrm{MeV}$ (soft-error sensitive range) [35]. For each neutron energy step, 10000 strike events are simulated to obtain the average energy deposition and charge ionization. Based on the neutron spectrum integration, 2.06 times enhancement of charge deposition is observed for InAs compared to $\mathrm{Si}$, which is applied to the following SER evaluation.

\section{B. SRAM and Combinational Logic SER}

Based on the critical LET and charge deposition analysis, the SRAM cell SER and logic SER are calculated based on (1) and (2) discussed in the Section III, respectively. Fig. 20(b) and (c) shows the relative SRAM SER and logic SER for Si FinFET, III-V FinFET and HTFET with the voltage scaling, respectively. HTFET shows superior soft-error resilience for voltage range from $0.3 \mathrm{~V}$ to $0.6 \mathrm{~V}$ for both SRAM and logic. 5 times reduction of SRAM cell SER and 30 times reduction of logic SER are obtained at $0.3 \mathrm{~V}$ for HTFET compared to Si FinFET. This improvement is contributed from both the improved critical LET and latching window masking effect at low voltage because of steep switching. III-V FinFET shows different SER performance for SRAM cell and logic compared to Si FinFET. For SRAM cell, III-V FinFET SRAM shows overall high SER due to both the charge deposition and collection enhancement, but with a shallower slope with the voltage scaling than Si FinFET SRAM due to the drive current improvement at low $\mathrm{V}_{\mathrm{DD}}$. As shown in Fig. 20(c), III-V FinFET logic shows lower SER below $0.5 \mathrm{~V}$ compared to $\mathrm{Si}$ FinFET logic. The combinational logic SER relates to both the critical LET and the latching probability, $w / c$. At high $V_{D D}$, the critical LET and charge deposition enhancement dominates the logic SER, where the latching window is comparable for III-V and Si FinFET at high drive current. At low $\mathrm{V}_{\mathrm{DD}}$, the latching window increases significantly for Si FinFET at near-threshold operation, which increases the error latched probability as well as the logic SER.

\section{CONCLUSION}

Sea-level radiation-induced soft error has been evaluated for Si FinFET, III-V (InAs) FinFET and III-V (GaSb as source/ InAs as channel and drain) HTFET using device to circuit simulation. The device simulation shows that the asymmetrical source/drain doping induced ambipolar transport in HTFET greatly reduces the minority carrier storage in the channel, 
which significantly reduces the bipolar gain effect and the charge collection as well as the transient duration. The III-V FinFET shows enhanced charge collection due to the mobility enhancement compared to Si FinFET. Based on the device simulation results, the SER based on critical LET extraction has been evaluated for SRAM cell and combinational logic, respectively, considering the charge deposition enhancement in III-V material with Geant4 simulation. Comparing Si and III-V FinFETs, III-V FinFET shows increased charge deposition due to lower ionization energy, which increases the SER for SRAM cells for all evaluated $V_{D D}$. The logic SER evaluation shows III-V FinFET has lower SER than Si FinFET below $0.5 \mathrm{~V}$, which results from the reduced latching probability at low voltage due to the improved latching window masking effect. Compared to $\mathrm{Si}$ and III-V FinFETs, HTFET shows superior radiation resilience over both $\mathrm{Si}$ and III-V FinFET with the voltage range of $0.3 \mathrm{~V}$ to $0.6 \mathrm{~V}$ for both SRAM and logic. This fundamental advantage stems from the bipolar gain reduction, the on-state enhanced Miller capacitance effect and the improved latching window masking. The significant SER reduction at low supply voltage makes III-V HTFET a promising candidate for radiation resilient ultra-low power applications.

\section{ACKNOWLEDGMENT}

The authors would like to thank Dr. K. Unlu and Dr. D. Sahin from the Department of Nuclear Engineering, The Pennsylvania State University, University Park, PA, USA for their help on Geant4 simulation.

\section{REFERENCES}

[1] P. Hazucha, T. Karnik, J. Maiz, S. Walstra, B. Bloechel, J. Tschanz, G. Dermer, S. Hareland, P. Armstrong, and S. Borkar, "Neutron soft error rate measurements in a 90-nm CMOS process and scaling trends in SRAM from 0.25-um to 90-nm generation," in IEDM Tech. Dig., 2003, pp. 21.5.1-21.5.4.

[2] S. Borkar, "Designing reliable systems from unreliable components: The challenges of transistor variability and degradation," IEEE Micro, vol. 25, no. 6, pp. 10-16, Nov./Dec. 2005.

[3] H. Kaul, M. Anders, S. Hsu, A. Agarwal, R. Krishnamurthy, and S. Borkar, "Near-Threshold Voltage (NTV) design-opportunities and challenges," in Proc. ACM/IEEE DAC, Jun. 2012, pp. 1149-1153.

[4] N. Seifert, P. Slankard, M. Kirsch, B. Narasimham, V. Zia, C. Brookreson, A. Vo, S. Mitra, B. Gill, and J. Maiz, "Radiation-induced soft error rates of advanced CMOS bulk devices," in Proc. IEEE Int. Rel. Phys. Symp., 2006, pp. 217-225.

[5] S. Borkar, T. Karnik, and V. De, "Design and reliability challenges in nanometer technologies," in Proc. 41st ACM/IEEE DAC, Jul. 2004, p. 75.

[6] L. Wei, S. Oh, and H.-S. P. Wong, "Performance benchmarks for Si, III-V, TFET, and carbon nanotube FET-re-thinking the technology assessment methodology for complementary logic applications," in IEDM Tech. Dig., Dec. 2010, pp. 391-394.

[7] A. C. Seabaugh and Q. Zhang, "Low-voltage tunnel transistors for beyond CMOS logic," Proc. IEEE, vol. 98, no. 12, pp. 2095-2110, Dec. 2010.

[8] D. K. Mohata, R. Bijesh, S. Mujumdar, C. Eaton, R. Engel-Herbert, T. Mayer, V. Narayanan, J. Fastenau, D. Loubychev, A. Liu, and S. Datta, "Demonstration of MOSFET-like on-current performance in arsenide/antimonide tunnel FETs with staggered hetero-junctions for $300 \mathrm{mV}$ logic applications," in Proc. IEEE IEDM, Dec. 2011, pp. 33.5.1-33.5.4.

[9] D. K. Mohata, R. Bijesh, Y. Zhu, M. K. Hudait, R. Southwick, Z. Chbili, D. Gundlach, J. Suehle, J. M. Fastenau, D. Loubychev, A. K. Liu, T. S. Mayer, V. Narayanan, and S. Datta, "Demonstration of improved heteroepitaxy, scaled gate stack and reduced interface states enabling heterojunction Tunnel FETs with high drive current and high on-off ratio," in Proc. Symp. VLSIT, Jun. 2012, pp. 53-54.
[10] V. Saripalli, J. P. Kulkarni, N. Vijaykrishnan, and S. Datta, "Variationtolerant ultra-low power heterojunction tunnel FET SRAM design," in Proc. IEEE/ACM Int. Symp. NANOARCH, San Diego, CA, USA, Jun. 2011, pp. 45-52.

[11] H. Liu, D. K. Mohata, A. Nidhi, V. Saripalli, V. Narayanan, and S. Datta, "Exploration of vertical MOSFET and tunnel FET device architecture for sub 10nm node applications," in Proc. IEEE DRC, Jun. 2012, pp. 233-234.

[12] C. A. Klein, "Bandgap dependence and related features of radiation ionization energies in semiconductors," J. Appl. Phys., vol. 39, no. 4, pp. 2029-2038, Mar. 1968.

[13] T. R. Weatherford and W. T. Anderson, Jr, "Historical perspective on radiation effects in III-V devices," IEEE Trans. Nucl. Sci., vol. 50, no. 3, pp. 704-710, Jun. 2003.

[14] D. McMorrow, J. B. Boos, A. R. Knudson, W. T. Lotshaw, D. Park, J. S. Melinger, B. R. Bennett, A. Torres, V. Ferlet-Cavrois, J. E. Sauvestre, C. DHose, and O. Flament, "Transient response of III-V field-effect transistors to heavy-ion irradiation," IEEE Trans. Nucl. Sci., vol. 51, no. 6, pp. 3324-3331, Dec. 2004.

[15] D. McMorrow, V. Ferlet-Cavrois, P. Paillet, O. Duhamel, J. Baggio, J. Brad Boos, and J. S. Melinger, "Transient response of semiconductor electronics to ionizing radiation recent developments in charge-collection measurement," IEEE Trans. Nucl. Sci., vol. 54, no. 4, pp. 1010-1017, Aug. 2007.

[16] M. Raine, G. Hubert, M. Gaillardin, L. Artola, P. Paillet, S. Girard, J.-E. Sauvestre, and A. Bournel, "Impact of the radial ionization profile on SEE prediction for SOI transistors and SRAMs beyond the 32-nm technological node," IEEE Trans. Nucl. Sci., vol. 58, no. 3, pp. 840-847, Jun. 2011.

[17] TCAD Sentaurus Device Manual, Synopsys, Mountain View, CA, USA, 2010.

[18] K. Castellani-Coulié, D. Munteanu, J.-L. Autran, V. Ferlet-Cavrois, P. Paillet, and J. Baggio, "Simulation analysis of the bipolar amplification induced by heavy-ion irradiation in double-gate MOSFETs," IEEE Trans. Nucl. Sci., vol. 52, no. 6, pp. 2137-2143, Dec. 2005.

[19] K. Castellani-Coulié, D. Munteanu, J.-L. Autran, V. Ferlet-Cavrois, P. Paillet, and J. Baggio, "Characterization time analysis of 45-nm multigate transistors behavior under heavy ion irradiation by 3-D device simulation," IEEE Trans. Nucl. Sci., vol. 53, no. 6, pp. 3265-3269, Dec. 2006.

[20] Y.-P. Fang and A. S. Oates, "Neutron-induced charge collection simulation of bulk FinFET SRAMs compared with conventional planar SRAMs," IEEE Trans. Device Mater. Rel., vol. 11, no. 4, pp. 551-554, Dec. 2011

[21] N. Agrawal, V. Saripalli, V. Narayanan, Y. Kimura, R. Arghavani, and S. Datta, "Will strong quantum confinement effect limit low Vcc applications of III-V FinFETs," in Proc. IEEE DRC, Jun. 18-20, 2012, pp. 231-232.

[22] M. Luisier and G. Klimeck, "Performance comparisons of tunneling fieldeffect transistors made of InSb, carbon, and GaSb-InAs broken gap heterostructures," in Proc. IEEE IEDM, Dec. 2009, pp. 37.6.1-37.6.4.

[23] K. Castellani-Coulié, D. Munteanu, V. Ferlet-Cavrois, and J.-L. Autran, "Simulation analysis of the bipolar amplification in fully-depleted SOI technologies under heavy-ion irradiations," IEEE Trans. Nucl. Sci., vol. 52, no. 5, pp. 1474-1479, Oct. 2005.

[24] K. Castellani-Coulié, D. Munteanu, J.-L. Autran, V. Ferlet-Cavrois, P. Paillet, and J. Baggio, "Investigation of $30 \mathrm{~nm}$ gate-all-around MOSFET sensitivity to heavy ions: A 3-D simulation study," IEEE Trans. Nucl. Sci., vol. 53, no. 4, pp. 1950-1958, Aug. 2006.

[25] Geant4. [Online]. Available: http://geant4.web.cern.ch/geant4

[26] J. F. Ziegler, "Terrestrial cosmic ray intensities," IBM J. Res. Develop., vol. 42, no. 1, pp. 117-139, Jan. 1998.

[27] Cadence Virtuoso Spectre Circuit Simulator 2009.

[28] P. Hazucha and C. Svensson, "Impact of CMOS technology scaling on the atmospheric neutron soft error rate," IEEE Trans. Nucl. Sci., vol. 47, no. 6, pp. 2586-2594, Dec. 2000.

[29] R. Naseer, Y. Boulghassoul, J. Draper, S. DasGupta, and A. Witulski, "Critical charge characterization for soft error rate modeling in $90 \mathrm{~nm}$ SRAM," in Proc. IEEE ISCAS, May 2007, pp. 1879-1882.

[30] R. Ramanarayanan, V. Degalahal, R. Krishnan, J. S. Kim, V. Narayanan, Y. Xie, M. J. Irwin, and K. Unlu, "Modeling soft errors at the device and logic levels for combinational circuits," IEEE Trans. Dependable Secure Comput., vol. 6, no. 3, pp. 202-216, Jul./Sep. 2009.

[31] J. L. Leray, "Effects of atmospheric neutrons on devices, at sea level and in avionics embedded systems," Microelectron. Reliab., vol. 47, no. 9-11, pp. 1827-1835, Sep.-Nov. 2007. 
[32] S. Mookerjea, R. Krishnan, S. Datta, and V. Narayanan, "On enhanced miller capacitance in inter-band tunnel transistors," IEEE Electron Device Lett., vol. 30, no. 10, pp. 1102-1104, Oct. 2009.

[33] P. Roche and G. Gasiot, "Impacts of front-end and middle-end process modifications on terrestrial soft error rate," IEEE Trans. Device Mater. Rel., vol. 5, no. 3, pp. 382-396, Sep. 2005.

[34] M. J. Bellido-Diaz, J. Juan-Chico, A. J. Acosta, M. Valencia, and J. L. Huertas, "Logical modeling of delay degradation effect in static CMOS gates," Proc. Inst. Elect. Eng.—Circuits, Devices Syst., vol. 147, no. 2, pp. 107-117, Apr. 2000.

[35] E. Normand, "Single event effects in avionics," IEEE Trans. Nucl. Sci., vol. 43, no. 2, pp. 461-474, Apr. 1996.

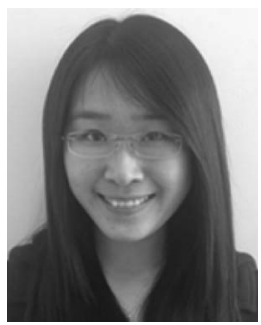

Huichu Liu (S'11) received the B.S. degree in microelectronics from Peking University, Beijing, China, in 2009. She is currently pursuing the Ph.D. degree in the department of Electrical Engineering, Pennsylvania State University, University Park, PA, USA.

Since 2009, she has been a Research Assistant with the Nanoelectronic Devices and Circuits Lab (NDCL) and Microsystem Design Lab (MDL) in Pennsylvania State University. From May 2011 to August 2011, she has been a summer intern at IBM T. J. Watson Research Center, Yorktown Heights, NY, USA. Her research interests include device-circuit interactions using emerging devices for ultralow power digital and analog/RF applications.

Ms. Liu was one of the winners of the IBM Ph.D. Fellowship Award from 2011 to 2012 Academic Year.

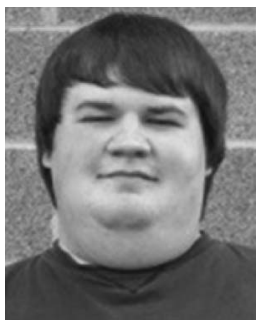

Matthew Cotter (S'09) received his B.S. degree in computer engineering from Penn State University, University Park, in 2008. He is currently a Ph.D. candidate in the department of Computer Science and Engineering, Pennsylvania State University, University Park, PA, USA.

Since 2009, he has been a Research Assistant with Microsystem Design Lab (MDL) in Pennsylvania State University. His research interests are focused on the applications of emerging device technologies in the acceleration and enhancement of neuromorphic hardware and similar systems.

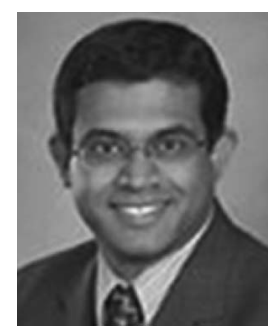

Suman Datta $\left(F^{\prime} 13\right)$ received the B.S. degree in electrical engineering from the Indian Institute of Technology, Kanpur, India, in 1995 and the Ph.D. degree in electrical and computer engineering from the University of Cincinnati, Cincinnati, OH, USA, in 1999.

From 1999 to 2007, he was a member of the Logic Technology Development Group, Intel Corporation. He was instrumental in the demonstration of indiumantimonide-based quantum-well transistors operating at room temperature with a record energy delay product, the first experimental demonstration of metal gate plasmon screening and channel strain engineering in high- $\kappa /$ metal gate CMOS transistors, and the investigation of the transport properties in nonplanar "trigate transistors." In 2007, he was the Joseph Monkowski Associate Professor of electrical engineering with Pennsylvania State University, University Park, where he is currently a professor of electrical engineering. He is the holder of more than 130 U.S. patents. His group is exploring new materials and novel device architecture for CMOS enhancement and replacement for future energy-efficient computing applications.

Dr. Datta is a Distinguished Lecturer of the IEEE Electron Devices Society.

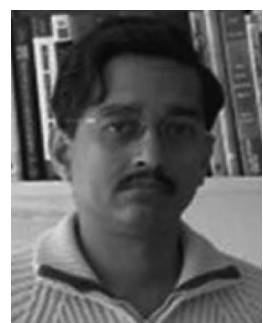

Vijaykrishnan Narayanan (F'11) received the B.S. degree in computer science and engineering from University of Madras, India, in 1993 and the Ph.D. in computer science and engineering from the University of South Florida, Tampa, USA, in 1998.

$\mathrm{He}$ is a professor of Computer Science \& Engineering and Electrical Engineering at Pennsylvania State University. His research interests include power-aware and reliable systems, embedded systems, nanoscale devices and interactions with system architectures, reconfigurable systems, computer architectures, network-on-chips, domain-specific computing.

Dr. Narayanan has received several awards including the Penn State Engineering Society Outstanding Research Award in 2006, IEEE CAS VLSI Transactions Best Paper Award in 2002, the Penn State CSE Faculty Teaching Award in 2002, the ACM SIGDA outstanding new faculty award in 2000 , Upsilon Pi Epsilon award for academic excellence in 1997, the IEEE Computer Society Richard E. Merwin Award in 1996 and the University of Madras first rank in Computer Science and Engineering in 1993. He is currently the editor-in-chief of IEEE Transactions on Computer Aided Design of Integrated Circuits and Systems. He has received several certificates of appreciation for outstanding service from ACM and IEEE Computer Society. 